\title{
Exploring the potential for CAl implementation in interpreter training in Ukraine
}

\author{
Viacheslav Karaban ${ }^{1}$, Anna Karaban ${ }^{2}$ \\ ${ }^{1}$ Department of the Theory and Practice of Translation from English, 01033 Taras Shevchenko \\ National University of Kyiv, Kyiv, Ukraine \\ ${ }^{2}$ Department of English Philology and Cross-cultural Communication, 01033 Taras Shevchenko \\ National University of Kyiv, Kyiv, Ukraine
}

\begin{abstract}
The article purports to assess the potential of the current European training in computer-assisted interpreting and associated technological competence development for making the corresponding technological turn in Ukraine's tertiary interpreter education based on a narrative review. Discussed are the issues of the technological turn in interpreting and its teaching, the corresponding awareness of educators and interpreters, the role of computer-assisted interpreting (CAI) tools in interpreting activities and training, the related challenges faced by interpreters and interpreters' training, and how Ukraine compares in this with the developments in the EU. Some problems are dwelt upon of COVID-propelled interpreting digitalization. Conclusions imply that Ukraine must undertake quickly such a technological turn not to lag behind the ongoing process of changes in interpreting and interpreter training.
\end{abstract}

\section{Introduction}

Interpreting is considered one of the most stressful jobs, exerting cognitive load and stress levels comparable to stockbrokers and flight dispatchers. It is somewhat anecdotal that the brain of a simultaneous interpreter during the interpreting session is similar to that of an individual on a stimulant drug. Nevertheless, the applications that could mitigate the like effects were not adequately addressed until quite recently. Another challenge is more evident from the academic perspective, while the emergence of computer-assisted interpreting tools started in the 2010s; they are neither industry standard nor widely implemented in the institutions that train future interpreters.

There is currently a lack of sufficient awareness about computer-aided tools for interpreters in Ukraine, especially compared to CAT ones. On a global scene, such a ratio could be explained by far larger industry demand in translation than interpreting, but this aside, there are other important reasons for such information vacuum in this field locally. One such factor is the global economy's impact, where Ukraine accounts for only a tiny fraction of the language services industry. Overall, developing countries have larger

${ }^{1}$ Corresponding author: v.karaban@knu.ua 
populations of non-English speakers, thus contributing to quite a different share of demand for interpreting versus translating jobs. This discrepancy means that while the pace of CAI advancement in developed countries corresponds to the demand, in developing economies like Ukraine, there is a greater share of interpreting jobs that could benefit from CAI progress. In other words, the lag in CAI development disproportionately affects developing countries and minor languages.

However, things are starting to change, CAI and CAI tools have experienced an upsurge of recent interest in the past decade, and, as Fantinuoli [1] promptly states, this area is far underresearched and underutilized by professionals and educators alike. For more than a decade, there has evolved a certain technological turn in this area, as noted by Fantinuoli [2] - there are already certain types of programs that assist interpreters at various stages. He attributes such technological turn to the progress in CAI software development and curricula inclusion thereof across Europe. Fantinuoli [2] believes the ongoing processes in CAI development and implementation can significantly transform how interpreting is done and drastically affect socio-economic and ergonomic prospects for interpreters. So if we are upon the global paradigm shift in interpreting, how does Ukraine not get left behind? What can be done to address the local challenges and walk in lockstep with the global interpreting community and training institutions?

Interpreters in Ukraine (like those worldwide) have benefited from CAT development, although, generally, CAT software is not entirely suitable for interpreters due to time constraints, software complexity, and lack of usability for interpreting workflows. Interpreter-assisting software began developing less than 15 years ago, and it is already changing the way interpreters work and showing potential to make a real difference in the field. Previously, interpreters performed their tasks with tools such as an interpreter console, an electronic panel used in the interpreter's booth that allows translators to switch languages, connect a headset, adjust volume, control microphones, etc. With CAT development, it became possible to utilize glossaries to help learn words and phrases, understand concepts and problems, and speed up access to data from the target language. Furthermore, now we are witnessing the emergence of interpretation platforms, i.e., technological systems that are specifically designed to aid the interpreting process, which allow interpreters to prepare for the job in advance, assist with the live interpreting, and can be useful in further processing of the speaker's speech. So with Ukrainian translators and translation students already benefiting from CAT tools, can interpreters and interpreting students in Ukraine get a leg up in their professions through CAI tools?

\section{Methods}

To address the issue of the technological component of interpreter training in Ukraine, it is best to approach the problem through literature review methodology. As the problem itself is four-fold, understanding technological competence of the interpreter, availability of tools for technological assistance of the interpreter and computer-assisted interpreter training, as well as the impact of post-COVID changes on the interpreting industry and training, it is more pragmatic to lay the groundwork in these aspects separately and consecutively through an extensive narrative review. Such review will help identify the gaps in interpreter training in Ukraine and establish available means of redressing such gaps by implementing the world's best practices in interpreter training as well as instructor education through familiarizing with such tools and evaluation of the fit of such tools for the Ukrainian language paired interpreters.

The following methods and research steps have been used to do the present narrative review: first, two research questions have been formulated to elucidate the issues in the 
development in CAI tools and interpreter training in the world for their use in interpreter training in Ukraine; best practices of Western universities on the forefront of employing CAI tools in training interpreters were observed. The paper's objectives were set as presenting an overview of the scholarly literature on CAI tools and interpreting training at tertiary educational institutions using them in the teaching process. The following steps were taken to conduct the narrative review: the extant relevant literature was screened within articles, journals, monographs, and research databases for their inclusion into the literature review. After that, the quality of primary studies has been assessed, and they have been chosen for their review. Relevant data have been extracted, compared, and analyzed to present and assess the suitability of the most important findings and solutions for applying them in designing a relevant syllabus of a course in interpreter training in Ukraine.

The materials for this review are published articles and books on CAI tools and their use in interpreter training in Interpreting Studies. The materials have been limited by the last decade when the research in the above area has gained momentum. The purpose of this literature review is to report the current knowledge on the topics at issue and present a summary on previously published research to place this information into the perspective of applying the conclusions in interpreter training in Ukraine.

\section{Results}

\subsection{The potential of improving technological competence in interpreter training}

The first potentiality for bridging the interpreter-training gap between Ukraine and the global community can be first approached through aligning the understanding of what interpreting competence is within the interpreter training programs.

One of the signs of the technological turn in interpreting is whether scholars in Interpreting Studies mention a requirement of technological competence among the interpreter's competences. Works on the technological competence in translation training predominate compared with research into the interpreter's technological skills. Not all papers on the latter published after 2015 mention, let alone discuss, any interpreter's technological competence. For example, in their characteristic of interpreting competence scales, Wang et al. [3] among their many parameters (interpretive strategies, interpreting knowledge, etc.) do not mention any technological skills. The interpreter's technological competence is not mentioned in the following papers on similar subjects $[4,5,6]$.

Some researchers are reluctant to include interpreters' technological competence into professional requirements under the pretext that nowadays, "technology has not yet reached the level of development required to provide human interpreter level of service, consecutive or simultaneous" [7]. However, it is worth reminding here with a view of computer-assisted interpreting (CAI) tools that Pym [8] emphasized as early as 2013 that technology is no longer just another add-on component.

In the EMCI syllabus for conference interpreting training at the postgraduate level, we only read that "Courses shall regularly expose students to new technologies in the work environment and include technology-mediated work scenarios such as remote interpreting and video-conference interpreting" [9]. This is too brief a statement on the interpreter's technological competence compared with requirements for the interpreters' technological competence from the Australian National Accreditation Authority for Translators and Interpreters: "Technological competency refers to Interpreting technology knowledge, skills in Interpreting through communication media, and the range of Information and Communication Technology (ICT) skills an interpreter must possess to prepare for and 
provide interpreting services, (i.e., Internet, software and hardware-based) ... Interpreting through communication media is now a standard form of work for many interpreters. This covers working through conference interpreting equipment, telephone interpreting, video or onscreen interpreting, use of portable audio equipment, and emerging areas of interpreting in multimedia contexts" [10].

De Céspedes [11] analyzes the place of technologies among several other competences and argues that technological competence is one of the competences translation courses graduates need to master to work in a professional setting. The interpreter's technological competence and instrumental needs were also presented in Oraki \& Tajvidi [12]. While technological competence is not well detailed and outlined within interpreter training programs, there is a well researched and documented area of technological assistance for interpreters compared to the equivalent translator training programs.

\subsection{The potential for using CAI tools}

There is also some potential in utilizing computer-assisted interpreting tools in the interpreter training in higher education institutions in Ukraine. Technological tools involved in interpreting workflow usually can be classified according to the nature of the interpreter's participation or the stage of interpretation: computer-assisted interpreting tools, computer-assisted interpreting training tools, and applications for remote interpretation. CAI tools include programs for helping interpreters prepare for interpreting events, assisting during the interpretation process, and managing the interpreting situation (or the interpreter's workplace), as well as ensuring the quality of interpretation.

During both preparation and live interpretation stages, CAI tools can help guarantee the quality of interpretation and the use of accurate terminology. Interpretation automation tools can reduce errors, optimize workflows, increase productivity, and improve quality when performing tasks. Most CAI tools incorporate glossary management, which assists professionals before, during, and after the event in preparing for tasks by providing a database of supporting materials, tools for retrieving, memorizing, understanding terminology, and the ability to synthesize documents instantly. It also assists the interpreter by providing access to multilingual glossaries and searching for equivalents in the target language if translators cannot remember specific terms. Searching for terms can be manual or automatic (using voice recognition). Finally, it supports the interpreter after the interpreting event through linguistic asset management, translation quality assessment, and reporting.

Although the European Commission's Directorate-General for Interpretation strategic plan for 2020-2024 [13] acknowledges the progress language technologies have made, it does not deem machine interpreting a threat. Nevertheless, they are working on Interpreters' Digital Toolbox, which by description is very akin to existing CAI tools, as it will be designed both to assist in the preparation stage through terminology extraction tools and live interpreting stage through integrated terminology databases and glossaries with a prospect of speech recognition integration at a later stage. Such strategies move the CAI tools to the forefront of interpreter training and a gradual shift of interpreters into a more digital workspace. European Commission's Directorate-General for Interpretation [14] also makes their focus AI automation of preparation stage tasks such as terminology extraction and management, shifting conference interpreting into cloud platforms, speech recognition, and automated transcription as well as acknowledging the emergence and increase in importance in hybrid modes of interpreting (inclusion of virtual component). The latter anticipated the European Commission's Directorate-General's Interpretation [13] central focus on the virtual and hybrid conferencing due to COVID and post-COVID realities. 
Among already existing CAI tools, which already provide the services the Interpreters' Digital Toolbox of the European Commission will be designed for, one cannot but mention several groups already available for interpreter assistance. First, terminology management tools for interpreting (Flashterm, InterpretBank, Interpreter's Help, Intragloss, Terminus, Lookup, Interplex, and Glossary Assistant) take into account the specifics of the interpreter's work such as limited time to search for translations of terms, etc. and exist for various operating systems, browsers and tablets as interpreters have a more dynamic workspace compared to translators. While corpora and parallel concordance dictionaries (Linguee, Glosbe) are similar in how terminology can be assessed and extracted, the latter show greater promise for future integration in interpreting systems as they already can provide assistance not only in preparatory stages but also in live sessions.

Speech recognition tools that are not necessarily designed for interpreters but add value to the experience (Dragon Naturally Speaking, Google Speech-to-Text, Google translate App speech transcription) are complemented by the proliferation of personal digital assistants that have voice recognition and notation capabilities (Siri, Amazon Alexa, Google Assistant). As of recent, closed-captioning services on streaming/video conferencing services (subtitling on Skype, closed-captioning on Youtube and Google Meet, live transcription on Zoom) experienced a boost with the rise of VRI. Such tools of this variety allow alleviating anxiety and stresses in live conference interpretation settings, serving as an "insurance" of sorts for the interpreter to refer to if the information was lost in the process.

Automatic translation tools, including AI/machine translation (Google Translate, DeepL, Bing), though not designed for interpreters and can better assist translators, has potential in combination with speech recognition by putting forward a provisional translation of transcribed speech in a live session where the interpreter at their discretion can choose whether to use the suggested translation. Also, VRI settings can potentially function as virtually self-sufficient speech to text translation.

Integrated systems, designed by and for interpreters already incorporate automatic speech recognition and terminology extraction in one interface (InterpretBank, CASSIS), although for now have limited capabilities in terms of languages, they show great progress CAI tools have made in the decade and potential for offering such integrated solution for a wider audience of interpreters.

Given the importance of the above programs for training interpreters in their study courses, it is necessary to acquaint students in detail and train them to work effectively with them. Computer dictionaries and terminology and abbreviation management programs are essential for interpreters.

\subsection{Challenges in taking advantages of CAl potential}

In order to utilize the fool potential of such tools, it is important to acknowledge the challenges, as the practicality of such tools is a subject of discussion in the community as making individual tools as well as integrated systems viable in the industry they have to add more than detract; Desmet et al. [15] state that without ergonomic design and presentation such tools could induce greater stress in the form of cognitive load.

Prandi [16] in her research addresses the issue of cognitive load, stating that in her study projections, cognitive load with the use of CAI is expected "to be higher during SI with CAI than during traditional SI but to be lower for CAI tools such as InterpretBank than for traditional terminology management solutions like Word and Excel glossaries" [16], while at the same time having better outcomes in the accuracy of terminological equivalence through the use of CAI. 
The traditional interpreting types were for many decades the industry standard, and without specific training in CAI, professionals tended to view various applications (ranging from basic word processing tools to CAI platforms) as more of a distraction and additional stress factor rather than the assistance they were intended to be.

Corpas Pastor [17] describes the findings of her survey on the technology used by interpreters. The group of consecutive interpreters had a larger share of users of various technologies assisting in interpreting - $79 \%$ compared to simultaneous interpreters $-69 \%$, while this share was significantly lower in liaison interpreting $-53 \%$, whisper interpreting - approximately $40 \%$, and $20 \%$ for sight interpreting. The same survey showed a higher interest in online tools $(57.89 \%)$ than computer applications $(34.21 \%)$ and a generally optimistic outlook on such tools. A more recent paper by Gaber et al. [18] broaches a very topical issue of ad hoc terminology extraction and ad hoc corpus compilation based on automatic speech recognition in video-recorded speeches. With the growth of VRI, a similar approach could prove to be instrumental in this area, as video conferencing platforms allow for the integration of the speech recognition tools discussed in the research.

Wang and Wang [19], in their research, conducted a survey which is very telling of the sentiment in the industry with one-third of the participants not noticing an increase in their performance (which there was) and $80 \%$ thinking that the CAI "tool is a distraction." The researchers state that this distraction was verbalized as a hindrance in attention management that goes in line with Prandi's [16] observations of the increase in cognitive load using CAI. $90 \%$ of participants were optimistic about the potential of the tool. In the same study [16], the minority of participants found the biggest benefit of CAI in relieving the stress of note-taking, yet $90 \%$ stated that long paragraphs of generated notes were not user friendly and not conducive to referencing the omitted information. Will [20], in his overview of CAI use, references numerous studies attesting to disinclination in the community to utilize CAI due to attention sharing leading to a potential increase of cognitive load.

A survey on the use of terminology extraction tools for interpreting purposes conducted by Goldsmith [21] suggests that existing terminology programs require more follow-up work and further development to better suit the needs of working interpreters. The demand was most marked in the possibility of extracting multi-word terms and their equivalents; context and continuous synchronous scrolling, manual input; extraction of unknown terms, multilingual extractions as noise minimization, external resource integration, and filtering. Moreover, this study goes in line with previous studies regarding the apprehensiveness of technology application in interpreting communities. Whereas the participants of the survey themself used terminology extraction CAI tools regularly, their view of their colleagues was more cautious - professional curiosity but apprehensiveness of unwillingness to utilize such tools. Goldsmith [21] also provided a review of tablet-based interpretation technologies in A comparative user evaluation of tablets and tools for consecutive interpreters.

From a technological perspective, the attitudes and technical problems associated with remote interpreting and acceptance of using CAI will gradually change. While the research from the 2000s still is very indicative of the current state, it predates the notion of digital citizenship. In her doctoral thesis, Darden [22] gives a comprehensive account of interpreter training to develop interpreting capabilities in the digital setting. Modern educators are already familiar with interpreter-supporting applications; however, the results of this study confirm serious shortcomings in resource and literature availability as well as institutional support in CAI training as part of the acquisition of digital citizenship skills as expressed in the interpreter educator communities.

Prandi [23] thoroughly addresses the current state of CAI training in interpreter training programs in Europe; the study's survey showed all respondents actively include CAI in their training, with the majority of the institutions having a separate CAI focused subject. In 
the same paper, Prandi [23] reiterates a common sentiment concerning CAI use by experienced translators, namely "a lack of interest, which could be explained by the fact that experienced interpreters usually already have their terminology management system and therefore do not update their knowledge in this field or try out new tools or systems."

Mellinger [24] advocates for process-oriented research in this field, specifically the potential of examination of professional setting and workflow in this setting, focusing on physiological and psychological data. At the same time, he draws attention to cognitive ergonomics in computer-mediated environments, which become more relevant not only regarding CAI use but in the current climate, driving interpreters to work in computermediated environments. In the aforementioned paper, Mellinger [24] underlines productoriented research as its role and place, i.e., the analysis of quality, agency, etc., it does not help address cognitive processes during interpreting tasks, which is directly correlated with CAIT as training based on the product-oriented obtained data while increases awareness in such technologies among trainees and allows working under assumptions about the cognitive processes, it does not directly help them approach matters like cognitive load, shared attention, etc.

\subsection{The potential of computer-assisted interpreter training and training in tools for computer-assisted interpreting}

When we next turn to the feasibility of application of CAI and CAIT tools in interpreter training classroom, we cannot but face the fact that the familiarity with the CAI tools is one of the biggest obstacles in making them an asset as opposed to added strain, interpreter training with such solutions should take priority in academic institutions. Although the literature on this matter is quite extensive, both theoretical and empirical papers manage to encompass enough specifics not to leave blind spots for the curious eye, there are neither reference materials nor student manuals.

De Céspedes [25] states that although machine translation and automation processes in the industry pose a degree of uncertainty for translator training, it is creativity, decisionmaking, and personability that will make the profession viable in the future. This can be extrapolated to interpreting, whereas automation in interpretation has not yet reached the heights of those in translation, these same variables will make human interpreters competitive in the future. All this means that technological advances will also give human interpreters an edge to fully automated interpreting systems if adopted in training stages. At the same time, interpreter training has been "the ugly stepsister" in training institutions, as Quist [26] points in her review to an imbalance in translator and interpreter training, with the former being favored over the latter at the same time observing that translation training almost reached the point of unwelcoming change and adjusting the priorities.

Back at the very beginnings of the 2010s, Tripepi Winteringham [27] argued the importance of utilizing technological advances in practice; now, some researchers argue the application of such advancements in training. Some studies [28, 29] underlined the importance of ICT in education in the field of translation; others [30] extended this statement to the field of interpreter training. The latter research argued that implementing ICT in interpreter training yielded statistically significant improvement information of competences for consecutive interpretation. More recent studies show that this call to action has found its realization in various training projects; accordingly, Sandrelli [31] names IVY (Interpreting in Virtual Reality) and Melissi as such modern immersive training experiences. Sandrelli [32, 33, 34] also underlines the importance of ICT and CAIT in interpreter training, with a focus on integrated immersive platforms, which resulted in the design of such trainer BlackBox, focusing on training live interpreter sessions traditionally. Modern versions of such tools by Melissi overlook the potential to include CAI in 
interpreter training, 'drilling' traditional approach into interpreting students, which later leads to apprehensive use of CAI. Qualitative data provided by Deysel \& Lesch [35] indicates that self-assessment of using this CAIT tool revealed a better understanding of interpreting the performance of the users. Thus Deysel \& Lesch [35] highlight the consequence of ICT use in interpreter training classrooms, or, as Fantinuoli [1] puts it, "preparing the profession to successfully face the upcoming technological turn". Yet, as Atabekova et al. [36] point out, "digital support of interpreting" is not adequately reflected in modern curricula.

\subsection{The potential of COVID-propelled digitalization of interpreting}

The final potentiality is the direct consequence of the challenge that interpreters worldwide faced during the pandemic. Considering a more extensive interest in CAI in the recent decade, it cannot be overlooked that COVID-19 has changed the world forever. The way business is conducted has changed forever, language services included, from the growing importance of remote interpreting [37] to an increase in telehealth interpreting. According to CSA Research [38], languages services not only have seen a decrease up to $55 \%$ but also account for a widespread view that the pandemic changed the market temporarily $(56 \%)$ to permanently $(41 \%)$ while showing an uptick in demand in translation for healthcare, medical, scientific, service and government industries and a decrease in leisure, event, and transport industries. At the same time, the report states: "LSPs on average still have less than half of their staff working in-office than they did before the pandemic $(35 \%$ before versus $77 \%$ now). Furthermore, LSPs do not plan to have the same level of in-office staff even after the pandemic is over - that number is down to $57 \%$ after the pandemic versus $77 \%$ before" [38]. The pandemic posed many challenges for most industries, but language service providers were also underprepared for the circumstances. A direct outcome of the pandemic is the rise in RSI (remote simultaneous interpreting) and VRI (video remote interpreting), both of which rely quite heavily on technology and are one of the biggest beneficiaries in the area of CAI. While research in this field has experienced a revival in the second part of the last decade, no one could have predicted the pandemic and the shift in the mode of interpreting to be done. The biggest share of pre-COVID interpreting was conducted traditionally - in-person interpreting, and while RSI, VRI, and other remote interpreting options existed, they were the fringe of the industry; thus, the skills needed to adjust were not trained across the industry, neither was the focus for these in the academia. In PwC's US Remote Work Survey [39], the overall trend of higher approval can be observed regarding the shift to remote work due to the pandemic. Even in the span of 6 months, there was an increase in enthusiasm in such shifts from $73 \%$ in June 2020 to $83 \%$ in the January 2021 report. PwC's survey [39] projects the shift toward a hybrid model post-pandemic and the change in the office role. This means language services industries could also expect a gradual change to more positive attitudes to remote work. While the initial shock of lockdowns exacerbated the stresses of familiarizing themselves with a new way to conduct business, translators and interpreters who grew more accustomed to remote work might develop a better outlook on it even in the post-COVID world.

Such sudden encumbrance to adopt new technology did not come without consequence, and just like CAI use has the potential to lead to an increase in cognitive load, remote interpreting also is rife with such side effects. Moser-Mercer's survey demonstrated that simultaneous interpreters faced with virtual interpreting platforms had "the additional challenge of "retrofitting the process" to overcome deficiencies created by the new environment," while the researcher questions even the cognitive capabilities for future interpreters to overcome such a challenge [40]. Braun [41] posits that remote interpreting could result in a "more rapid increase in fatigue" during the process while still showing a 
potential for adaptability of the interpreter. This fatigue could be explained through unfamiliar interpreting settings and challenges of remote interpreting (sound quality, turntaking, coordination, etc.), and the drastic shift to the remote workplace in 2020 due to the ongoing pandemic corroborated such findings on a mass scale, with terms like "zoom fatigue" entering the mainstream lexicon. This phenomenon has already been studied as a spatial problem [42] and focus issue [43], which are part and parcel of computer-mediated communication. Some solutions to how mental issues could be addressed and assault on cognitive function minimized were established. The challenge interpreters faced in remote interpreting settings - that of adjusting to an unfamiliar setting - could be addressed with more exposure to such environments, further training in video conferencing settings.

However, the boom in video conferencing platform due to the pandemic resulted in the majority of such services offering closed-captioning and subtitling (the built-in function in GSuite's Google Meet, the third party closed captioning integration into Zoom, closed captioning and subtitling features in Skype, etc.), this alone takes care of one of speech recognition element of CAI. Such technologies could be a fierce competition for CAI tools (including integrated tools) as video conferencing giants or tech industry leaders like Google either already have a stake in machine translation services and speech recognition or have all the right elements to capture the market. With the speech recognition and notetaking already provided by a video conferencing platform, CAI tools providing exclusively terminology management might not be enough actually to relieve the stress of interpreting. CAI applications have to evolve fast with more integrated capabilities designed to address interpreters' needs in virtual settings. The tech giants can lose interest in their side projects, even in the language services industry - Google Translator Toolkit was abandoned in 2019, but only because of the fierce competition in the field. CAI tools at the moment do not have integrated solutions for video interpreting, nor were they designed to mitigate the stresses associated with remote interpreting. All CAI tools are designed to assist traditional interpretation rather than remote interpreting, which is a gap in the market that will not be open for long as RI is advancing to become an industry standard.

\section{Discussion}

So what is the potential for transplanting best practices in interpreter training into Ukrainian higher education institutions? Aside from the universal challenges in new technology adoption, when we approach the CAI challenges within a local setting, we can establish at least several major contributing obstacles for a Ukrainian interpreter. The first is the underdeveloped infrastructure within the speech recognition field, particularly affecting minor languages, more so in countries where native speakers of a minor language are bilingually competent in another language (for Ukraine, it is the prevalence of Russian), which dampens the drive for accuracy in Ukrainian language recognition when a more accurate Russian language tool is already available. It has a bearing on one more barrier for greater reliance on software support while interpreting - the lower quality of machine translation to and from minor languages. While like in the previous explanation, Ukrainian speakers can get by with machine translation to and from Russian (which is more accurate due to being a major international language) in everyday communication, as far as professionals in the sphere of interpreting are concerned - lack of advances in the field with reference to the Ukrainian language puts them at a disadvantage compared to interpreters with major languages. The final essential hindrance is fewer freely available concordances as well as institutional TMs and terminology databases with the Ukrainian language; as it is not a language of intergovernmental structures (unlike those of EU), neither an international 
language, translators are a lot more reluctant to upload their TMs and terminology databases for public cloud-based services available.

However, all these issues can be addressed or at least eased to a substantial degree. For instance, the first consideration can be tackled through a concerted effort of interpreters, translators, and their professional unions as well as academic institutions training future professionals in the fields by supporting local speech recognition developers by supplying segmented and transcribed data from educational media or recordings of completed jobs/projects; also, from an academic perspective through utilizing more local speech recognition applications, like Odrey, and educating the students on their use. The second obstacle poses more of a challenge: while the lower quality of machine translation is what keeps translators and interpreters in business, bettering the machine output would greatly facilitate the work of a simultaneous interpreter, supplementing their reliance on machine translation with terminology management tools.

In the post-Soviet space, Ukraine in particular, empirical data on CAI application is currently unavailable. However, on screening publicly available curricula and syllabi of the institutions training translators and interpreters, one has yet to find a subject exclusively dedicated to studying CAI or even include CAI in more general translator/interpreter training syllabi. At the same time, although severely lagging behind the EU in CAT training, most Bachelor and Master training programs at Ukraine's universities include if not a separate dedicated CAT training subject, at least a significant allotment of hours to it. The interpreter training in the post-Soviet area could benefit from including such cutting edge technologies in the fields as used in the EU, and thus are remiss at not updating curricula and syllabi to include those. However, a common thread in research in this field is that weaknesses in interpreter training in this area are not for the lack of enthusiasm of educators as Prandi [16] and Deysel \& Lesch [35] observe. If specialized curricula were to be developed and the resources were to be popularized in the training institutions, a more standardized interpreter training approach could be found. While at the same time, educators in the post-Soviet space lack familiarity with such tools and literature on this subject, thus they would need to receive the like training themselves first to be able to train their students in CAI tools properly. Prandi mentions, "survey is quite representative of the European landscape, but further research is needed to expand this observation on a global scale" [16]. Further research in post-Soviet countries could shed more light on the gravity of CAI training shortcomings, in Ukraine in particular, which in turn may be conducive to looking into ways of rectifying the situation.

While, as mentioned before, at the moment, interpreters are somewhat reluctant to utilize CAI tools, there could be a reason not yet mentioned that could be valid for the postSoviet space, namely, financial constraints of the emerging economies. European developers of such software tend to neglect a common practice with CAT developers extensive cooperation with academic institutions (for example, academic licensing from SmartCAT, Memsource), allowing students (future interpreters) to familiarize themselves with the software, which is an established model in the digital space: providing a generous free service and in such a way training their potential customers to rely on their service. The generation currently entering interpreting training programs are digital natives who acquire digital citizenship skills faster than generations before, already pre-programmed to transitioning from generous demos and limited use free licenses to becoming paid customers. It could be crucial for popularizing and monetizing such tools among users from the developing economies, which would benefit the further advancement, expansion, and, finally, popularity of such software.

\section{Conclusion}


We witness a technological turn in interpreting and a certain surge in the development of CAI tools in the EU as well as an expanding wave of CAI teaching at western universities. All this is reflected in respective published articles and discussions on how to form a wider technological competence in would-be interpreters. On the contrary, we can state that at Ukraine's universities there is no sufficient awareness of the technological turn in interpreting that is taking place in the West. We must immediately address the lack of awareness of such a field as CAI and such tools in Ukraine through teaching, article circulation, reference, and calibration to the respective European syllabi. This avenue could include the publication of interpreter training manuals describing CAI tools and how to use them in interpreting. It is necessary to make interpreter trainers aware of the whole range of the available technological interpreter support and urge them to design and present teaching modules, video tutorials, and reference materials dealing with CAI tools. There should be a classroom focus on tools available for the Ukrainian language, namely parallel corpora and concordances, terminology extractors, and glossary management tools. At the same time, there should be monitoring of the availability of CAI tools that presently are languagebound and lack support for Ukrainian, i.e., speech recognition, automatic transcription, and integrated interpreter aiding systems.

With the boost in computer and, more specifically, video conferencing literacy due to COVID in Ukraine, there arises an opportunity to delve deeper into VRI training in its CAI capacity. So, it is crucial to develop syllabi considering the technological aspect of VRI and its potential for interpreter support and training. To offset the challenges for CAI and RI laid out in the paper, there is an opportunity to convert those into practical moments, e.g. treating time delay of video conferencing as an asset, getting students working on ways to utilize auto-subtitles from such services into interpreting aid, focusing on multitasking with parallel concordance lookup of auto-generated text until integrated systems become available for the Ukrainian language, thus working on adjusting students to increased cognitive load associated with the use of CAI.

The untapped potential addressed in the article testifies to a considerable lag of Ukrainian universities in the quality and level of interpreter training as far as technological backup is concerned. Their training programs should be reconsidered and renovated to consider the latest developments in the field of CAI and CAIT. It is the task of such leading universities as Taras Shevchenko National University in Kyiv, Vasil Karazin National University in Kharkiv, and Ivan Franko National University in Lviv, as well as the involvement of the Ukrainian Association of University Teachers of Translation and the Academy of Sciences of the Higher School of Ukraine, whose primary responsibility is to facilitate the improvement of training interpreters at higher educational institutions through closer cooperation with corresponding European universities, and last but not least the Ukrainian Association of Translators and Interpreters. Numerous translation and interpreting agencies with special technological resources for interpreting may well be interested in assisting Ukraine's universities in organizing internship interpreting practices employing CAI. To attract the attention of interpreter trainers to the necessity of a technological turn in interpreter teaching, Ukraine's universities must hold special international conferences and publish more theoretical and methodological articles dealing with CAI and its teaching. Ukrainian interpreter training universities must introduce special CAI modules into their syllabi of interpreting courses.

Finally, we must bring the attention of Ukrainian speech recognition developers to the CAI market; thus, by cooperating with interpreter training institutions, there could be potential to develop integrated CAI systems targeted at Ukrainian interpreters. The country's interpreter training institutions, in the meantime, can approach the European developers of CAI tools for educational licenses and/or training in the use of their tools. In general, it is difficult to predict how the speed of development of automated interpreting 
technologies will affect the training of interpreters and, in particular, change of course to technological training of interpreters. However, one thing is obvious - Ukraine is already behind in CAI literacy. If it is not addressed in the immediate future, anachronistically trained interpreters (thus inferior in performance and adaptability compared to CAI-trained professionals) might be easier prey for fully automated interpreting systems.

\section{References}

1. C. Fantinuoli. Interpreting and technology. Translation and Multilingual Natural Language Processing 11, 1-12 (2018)

2. C. Fantinuoli. Proceedings of the conference Übersetzen und Dolmetschen. 4, 334-354 (2019)

3. W. Wang, Y. Xu, B. Wang, and L. Mu. Front. Psychol. 11:481 (2020)

4. I. Horváth. The Modern Translator and Interpreter Book. 183-193 (2016)

5. I. Horváth. The Modern Translator and Interpreter Book. 233-242 (2016)

6. S. Kalina. Interpreters Newsl. 10, 3-33 (2000)

7. I. Horváth. Rev. Int. Lang. Mod. Appl. 7, 19-26 (2013)

8. A. Pym. Meta: J. Trad. 58(3), 487-503 (2013)

9. European Masters in Conference Interpreting. EMCI Quality Assurance Standards. https://www.emcinterpreting.org/emci/application/files/7815/2751/1862/EMCI_Qualit y_Assurance_Standards.pdf (2018)

10. National Accreditation Authority for Translators and Interpreters. NAATI Interpreter Certification: Knowledge, Skills and Attributes. https://www.naati.com.au/wpcontent/uploads/2020/01/Interpreter-KSA-Paper.pdf (2016)

11. R. De Cespedes. Optimización de las Competencias del Traductor e Intérprete: Nuevas Tecnologías-Procesos Cognitivos-Estrategias. 143-162 (2018)

12. A. Oraki, G. Tajvidi. Iran. J. Engl. Acad. Purp., 9(2), 42-56 (2020)

13. European Commission's Directorate-General for Interpretation. Strategic Plan 2020$2024 D G$ Interpretation. https://ec.europa.eu/info/system/files/scic_sp_20202024 en.pdf (2020)

14. European Commission's Directorate-General for Interpretation. New Technologies and Artificial Intelligence in the field of language and conference services. https://ec.europa.eu/education/knowledge-centreinterpretation/sites/kci/files/final host_paper_iamladp2019 en_version.pdf (2019)

15. B. Desmet, M. Vandierendonck, \& B. Defrancq. Interpreting and technology. Translation and Multilingual Natural Language Processing 11, 13-27 (2018)

16. B. Prandi. Interpreting and technology. Translation and Multilingual Natural Language Processing 11, 29-61 (2018)

17. G. Corpas Pastor. CTTTL E, 5, 157-182 (2018)

18. M. Gaber, G. Corpas Pastor, and A. Omer. TRANS: Rev. Trad. 24, 263-281 (2020)

19. X. Wang, C. Wang. Transletters. Int. J. Transl. Interpreting. 2, 109-139 (2019)

20. M. Will. ESSACHESS. J. Commun. Stud. 13(25), 37-71 (2020)

21. J. Goldsmith. Interdependenzen und Innovationen in Translation und Fachkommunikation: Interdependence and Innovation in Translation, Interpreting and Specialised Communication, 279-304 (2020) 
22. V. Darden. Walden Dissertations and Doctoral Studies. 7627. (2019)

23. B. Prandi. InTRAlinea. 2512 (2020)

24. C. D. Mellinger. Tradumàtica. 17, 33-44 (2019)

25. R. De Cespedes. Leb, Sprachen, 64(1), 103-121 (2019)

26. J. Quist. TranscUlturAl: J. Transl. Cult. Stud., 11(1), 145-146. (2019)

27. S. Tripepi Winteringham. Interpreters Newsl. 15, 87-99 (2010)

28. O. Ivanova. Procedia Soc. Behav. Sci., 231, 129-134 (2016)

29. I. Sikora, M. Walczyński. Transl. Comp. 2, 119-133 (2015)

30. E. V. Tikhonova, N. S Tereshkova. Procedia Soc. Behav. Sci., 154, 534-538 (2014)

31. A. Sandrelli. Routledge Encyclopedia of Interpreting Studies. 75-77 (2015)

32. A. Sandrelli. In MuTra 2005-Challenges of Multidimensional Translation: Conference Proceedings. (2007)

33. A. Sandrelli. MonTI. 2, 111-138 (2015)

34. A. Sandrelli, J. D. M. Jerez. Interpret. Transl. Train. 1(2), 269-303 (2007)

35. E. Deysel, H. Lesch. Interpreting and technology. Translation and Natural Language Processing 11, 61-90 (2018)

36. A. Atabekova, R. G. Gorbatenko, T. V., Shoustikova \& C. Valero-Garcés. J. Soc. Stud. Educ. Res., 9(3), 351-369 (2018).

37. The United Nations Department of Global Communications. Portraits: UN interpreters adapt to new work modes during COVID-19. https://www.un.org/en/coronavirus/portraits-un-interpreters-adapt-new-work-modesduring-covid-19 (2020)

38. CSA Research. COVID-19 LSP 3 Survey. https://csa-research.com/More/FeaturedContent/Leadership-Resources/LSP-3-Survey (2020)

39. PricewaterhouseCoopers. Business needs a tighter strategy for remote work. PwC. https://www.pwc.com/us/en/library/covid-19/us-remote-work-survey.html (2021)

40. B. Moser-Mercer. Meta: J. Trad. 50(2), 727-738 (2005)

41. S. Braun. Interpreting, 15(2), 200-228 (2013)

42. R. Nadler. Comput. Compos., 58, 102613 (2020)

43. S. Cranford. Matter, 3(3), 587-589 (2020) 